\title{
Research Article \\ Susceptibility of Mature Staphylococcus Biofilms to Chinese Herbal Decoction Sanhuang Jiedu: An In Vitro Study
}

\author{
Shaoe Zhang, Xiao Wang, Xiaotao Shi, and Honglue Tan \\ Henan Orthopedic Institute, Henan Luoyang Orthopedic-Traumatological Hospital (Henan Orthopedic Hospital), \\ Luoyang 471000, China \\ Correspondence should be addressed to Honglue Tan; hnlc.love@163.com
}

Received 13 April 2020; Accepted 18 August 2020; Published 28 September 2020

Academic Editor: Himanshu Garg

Copyright (c) 2020 Shaoe Zhang et al. This is an open access article distributed under the Creative Commons Attribution License, which permits unrestricted use, distribution, and reproduction in any medium, provided the original work is properly cited.

\begin{abstract}
Background. External socking and washing with the Chinese herbal Sanhuang Jiedu decoction (SHJD) can effectively control local limb infections with bone and implant exposure. However, the antibiofilm activities of this decoction in vitro have not yet been investigated. Therefore, the aim of this study was to examine the effects and characteristics of SHJD on the mature biofilms of multidrug-resistant staphylococci on a titanium surface. Methods. Biofilm-forming methicillin-resistant Staphylococcus epidermidis ATCC 35984 and S. aureus ATCC 43330, and non-biofilm-forming S. epidermidis ATCC 12228 were selected as the experimental strains. The mature biofilms were prepared on titanium surfaces. The five experimental groups were based on dilution concentrations (DC) of SHJD: the control group (biofilm incubated with $0.85 \% \mathrm{NaCl}$ solution), the SHJD (DC:1/8) group (initial SHJD solution was diluted 1/8), the SHJD (DC:1/4) group, the SHJD (DC:1/2) group, and the SHJD (DC:1/1) group (initial SHJD solution). The effects of SHJD on the mature biofilms were observed with the bacterial spread plate method, crystal violet (CV) staining, scanning electron microscopy, and confocal laser scanning microscopy. Results. After culture in tryptic soy broth for $72 \mathrm{~h}$, ATCC 43300 and ATCC 35984 produced mature biofilms and ATCC 12228 did not. The optical density value of ATCC 12228 was $0.11 \pm 0.02$, significantly lower than that of ATCC $35984(0.42 \pm 0.05)$ or ATCC 43300 $(0.41 \pm 0.03)(P<0.05)$. The mature biofilms of ATCC 43300 and ATCC 35984 clearly disintegrated when incubated for $12-24 \mathrm{~h}$ with SHJD (DC:1/1) or SHJD (DC:1/2), showing only scattered bacterial adhesion. In the SHJD (DC:1/4) group, although many residual bacterial colonies still clustered together, presenting a biofilm structure, it was very looser than that in the SHJD (DC:1/8) group in which the biofilm was similar to that in the control group. For ATCC 12228, only colony adhesion was observed, and the number of colonies decreased as the concentration of SHJD or the culture period increased. The quantitative results for the bacterial spread plate and CV staining showed significant differences between the SHJD groups $(P<0.05)$. Conclusion. SHJD has antibiofilm activity against multidrug-resistant Staphylococcus strains. It weakens or disrupts alreadyformed mature biofilms on titanium surfaces in a concentration- and incubation time-dependent manner.
\end{abstract}

\section{Introduction}

The management of refractory orthopedic-implantassociated infections is still challenging for surgeons because bacterial biofilms can form [1]. Biofilms are communities of cells within a self-produced extracellular matrix and are the commonest form of microbial life [2]. In the presence of this matrix, bacteria are highly resistant to antibiotics, with minimum inhibitory concentrations up to 1,000-fold higher than those of their planktonic forms [3]. Pathogenic bacterial biofilms can successfully evade the host's immune system and cannot be phagocytized and eliminated by polymorphonuclear leukocytes [4]. Therefore, the inhibition and removal of a bacterial biofilm from an infected area is an important procedure in the treatment of orthopedic infections.

The long-term application of systemic antibiotics is necessary for acute and chronic orthopedic infections associated with mature biofilms, together with local wound debridement [5]. However, the clinical reality is that refractory infections are always caused by multidrug-resistant (MDR) Staphylococcus epidermidis or S. aureus, and after the clinical susceptibility of the bacterium involved to antibiotics, 
vancomycin is usually the treatment of choice for biofilmassociated infections [6]. Unfortunately, the widespread use of vancomycin has led to the emergence of vancomycinresistant Staphylococcus strains [7]. Linezolid is a new type of antibacterial agent with broad-spectrum antibacterial activity against most common Gram-positive bacteria and has been very useful in the treatment of MDR Staphylococcus infections when vancomycin fails. However, safety concerns often limit its use [7]. Clinical reports have also indicated the existence of linezolomine-resistant staphylococci [8]. Therefore, based on the emergence of antibiotic resistance, adverse side effects, and a lack of new antibiotics, other treatment methods are urgently required for orthopedic-biofilmrelated infections caused by MDR bacteria.

Traditional Chinese medicine (TCM) has been used in the Chinese population to treat various infectious diseases for a long time, and many preparations have proven therapeutic effects [9-14]. According to the TCM theory, the Chinese herbal decoction Sanhuang Jiedu (SHJD), which is composed of Scutellaria baicalensis Georgi (Huang Qin), Coptidis rhizoma (Huang Lian), Cortex Phellodendri chinensis (Huang Bai), and so on, has heat-clearing and detoxifying functions. Based on the TCM theory, acute infection belongs to the "excess-heat syndrome"; the treatment of infection should be focused on the "heat-clearing." SHJD has the effect of clearing "excess-heat"; thus, its clinical application can control tissue infections. We have used this preparation to treat local limb infections with bone and implant exposure by local soaking and washing methods. The observation of a large number of cases has shown that local infections are significantly controlled and that the granulation tissue grows well, improving the condition of the wound tissue so that further treatment is possible [12-14]. However, there have been few in vitro studies of the antibiofilm activity of this preparation. Therefore, we used MDR S. aureus and S. epidermidis to investigate the effects and characteristics of SHJD on mature biofilms of these bacterial strains on a titanium surface.

\section{Materials and Methods}

2.1. Preparation of SHJD. The ingredients of SHJD decoction were purchased from Henan Luoyang Orthopedic-Traumatological Hospital (Luoyang, China) and included Scutellaria baicalensis Georgi (Huang Qin), Coptidis rhizoma (Huang Lian), and Cortex Phellodendri chinensis (Huang Bai) with $25 \mathrm{~g}$ of each dry herb, and Sophora flavescens (Ku Shen), Lonicerae japonicae (Jin Yin Hua), Forsythiae suspensa (Lian Qiao), Taraxacum mongolicum Hand.-Mazz.(Pu Gong Ying), Semen persicae (Tao Ren), Boswellia sacra (Ru Xiang), Commiphora myrrha (Mo Yao), and Radix glycyrrhizae (Gan Cao) with $20 \mathrm{~g}$ of each dry herb (Table 1). The aqueous extract of SHJD was prepared as follows. First, all the ingredients were placed into an earthenware pot containing $3000 \mathrm{~mL}$ of ultrapure water for about $30 \mathrm{~min}$, boiled with a strong fire, and then with a gentler fire about $30 \mathrm{~min}$ until the mixture was reduced to $1000 \mathrm{~mL}$. Second, the water extract was filtered through filter paper and evaporated under reduced pressure to a final concentration of $0.7 \mathrm{~g} / \mathrm{mL}$ based on the equivalent amount of the crude dry herb. Third, the water extract was sterilized by filtration through a $0.22 \mu \mathrm{m}$ filter. It was then added to a sterile $\mathrm{NaCl}$ solution $(0.85 \%)$ to a final SHJD concentration of $0.4 \mathrm{~g} / \mathrm{mL}$. The aqueous extract of SHJD was stored at $-20^{\circ} \mathrm{C}$ until use.

2.2. Preparation of Bacterial Strains. Three bacterial strains were used in this study: biofilm-forming MDR S. aureus (ATCC 43300), methicillin-resistant S. epidermidis (ATCC 35984), and non-biofilm-forming methicillin-sensitive $S$. epidermidis (ATCC 12228). The strains were thawed rapidly, used to inoculate tryptic soy agar (TSA; Shanghai Biotech Co., Ltd., China), and cultured statically at $37^{\circ} \mathrm{C}$ for $24 \mathrm{~h}$. A colony was picked from this agar and suspended in $10 \mathrm{~mL}$ of tryptic soy broth (TSB; BD Biosciences, Franklin Lakes, NJ) supplemented with $0.5 \%$ glucose in a $50 \mathrm{~mL}$ centrifuge tube, and incubated for $10 \mathrm{~h}$ at $37^{\circ} \mathrm{C}$ with agitation at $100 \mathrm{rpm}$. The aliquots $(25 \mu \mathrm{L})$ of this preculture were transferred into other sterile tubes containing $10 \mathrm{~mL}$ of TSB and incubated at $37^{\circ} \mathrm{C}$ for $14 \mathrm{~h}$, which was sufficient to ensure that the strains were at the end of the exponential phase of growth [15]. The bacteria in the suspensions were harvested by centrifugation for $5 \mathrm{~min}$ at $1000 \mathrm{rpm}$ (Sorvall TC6, Dulont, USA), and the precipitate was washed three times with $0.15 \mathrm{M}$ phosphate-buffered saline (PBS) to remove the remaining TSB. The pellet was resuspended in sterile PBS to an optical density $(\mathrm{OD})$ at $600 \mathrm{~nm}\left(\mathrm{OD}_{600}\right)$ of 0.490 , measured with a Synergy HT multidetection microplate spectrophotometer (BioTek, Winooski, VT), which corresponded to 109 CFUs (colony-forming units)/mL.

2.3. Mature Biofilms on Titanium Surfaces. To prepare the mature biofilms, sterile titanium discs $(14 \mathrm{~mm}$ in diameter and $1 \mathrm{~mm}$ thick) were placed into the wells of a 24-well plate (Costar3548, USA), with six wells allocated to each strain. Bacterial cells of strains ATCC 35984, ATCC 43300, and ATCC 12228 were diluted to a concentration of $10^{6} \mathrm{CFUs} / \mathrm{mL}$ in TSB, and $2 \mathrm{~mL}$ of each cell suspension was added to a well. After incubation for $72 \mathrm{~h}$ without agitation, the discs were rinsed three times with PBS to remove nonadherent cells and transferred to another sterile 24-well microtiter plate. The mature biofilms formed on the titanium surfaces were identified with crystal violet (CV) staining assay [2], confocal laser scanning microscopy (CLSM), and scanning electron microscope (SEM) [16].

2.4. Effect of SHJD Concentration on Mature Biofilms. The five experimental groups were based on dilution concentrations (DC) of SHJD: the control group (biofilm incubated with $0.85 \% \mathrm{NaCl}$ solution only), the SHJD (DC:1/8) group (initial SHJD solution was diluted 1/8), the SHJD (DC:1/4) group (initial SHJD solution was diluted 1/4), the SHJD (DC:1/2) group (initial SHJD solution was diluted 1/2), and the SHJD (DC:1/1) group (initial SHJD solution). Mature bacterial biofilms were prepared on titanium surfaces as described above. For each strain, an aliquot of $2 \mathrm{~mL}$ of $\mathrm{NaCl}$ and $\mathrm{NaCl}$ containing a different concentration of SHJD was dispensed into each well of the 24 -well microtiter plate containing a titanium disc covered with a mature bacterial biofilm, with six replicate wells for each group. The plates were 
TABLE 1: The composition of SHJD decoction.

\begin{tabular}{|c|c|c|c|}
\hline Chinese name & Latin name & Part used & Proportion (g) \\
\hline Huang Qin & Scutellaria baicalensis Georgi & Root & 25 \\
\hline Huang Lian & Coptidis rhizoma & Root & 25 \\
\hline Huang Bai & Cortex Phellodendri chinensis & Bark & 25 \\
\hline Ku Shen & Sophora flavescens & Rhizome & 20 \\
\hline Jin Yin Hua & Lonicera Japonica & Bud & 20 \\
\hline Lian Qiao & Forsythia suspensa & Fruit & 20 \\
\hline $\mathrm{Pu}$ Gong Ying & Taraxacum mongolicum hand.-Mazz. & Root & 20 \\
\hline Tao Ren & Semen persicae & Seed & 20 \\
\hline Ru Xiang & Boswellia sacra & Gummo-oleoresins & 20 \\
\hline Mo Yao & Commiphora myrrha & Gummo-oleoresins & 20 \\
\hline Gan Cao & Radix glycyrrhizae & Root & 20 \\
\hline
\end{tabular}

incubated under aerobic conditions with a low shaking rate of $100 \mathrm{rpm}$ for $24 \mathrm{~h}$. After incubation, the culture medium containing any planktonic bacteria was carefully removed from each well. The discs were transferred into another fresh 24-well plate and gently washed three times with sterile PBS to remove any loosely adherent bacteria. The residual biofilms of each strain on the titanium surfaces were analyzed with the following methods.

2.4.1. Crystal Violet (CV) Staining. The biofilms on the disc surfaces were analyzed with $\mathrm{CV}$ staining according to a previous report [2]. Glutaraldehyde solution $(2.5 \%, 2 \mathrm{~mL})$ was pipetted into each of the drained wells and allowed to stand for $10 \mathrm{~min}$ to fix the cells. The glutaraldehyde solution was then removed, and the wells were washed three times with PBS. The PBS was removed, and $2 \mathrm{~mL}$ of $0.1 \%(\mathrm{w} / \mathrm{v})$ aqueous $\mathrm{CV}$ solution was added to each well and incubated for $20 \mathrm{~min}$ at room temperature. The CV solution was discarded, and the wells were washed three times with PBS and air-dried for $12 \mathrm{~h}$ in the dark. The quantity of the biofilm was analyzed by adding $2 \mathrm{~mL}$ of $30 \%$ acetic acid to each well to dissolve the dye from the adherent cells (biofilm) for $30 \mathrm{~min}$. Then, $200 \mu \mathrm{L}$ of the dye solution was transferred into a 96-well microtiter plate, with the samples from one group assigned to a vertical row of the plate wells. The $\mathrm{OD}_{492}$ of each well was determined with a plate reader.

2.4.2. Spread Plate Method. The assay was performed according to a previous report [16]. After incubation for $24 \mathrm{~h}$ in different culture media, each titanium disc whose surface was covered with biofilm was lightly washed with PBS and transferred to a $10 \mathrm{~mL}$ glass tube containing $1 \mathrm{~mL}$ of PBS. The tubes were then placed in an ultrasonic bath (B3500S-MT, Branson Ultrasonics Co., Shanghai, China), and the bacteria or the biofilm on the discs were dislodged by ultrasonication ( $5 \mathrm{~min}$ ) at an operating power of $150 \mathrm{~W}$ and a frequency of $50 \mathrm{~Hz}$. Ultrasonication was followed by rapid vortex mixing (Vortex Genie 2, Scientific Industries, Bohemia, NY, USA) at maximum power for $1 \mathrm{~min}$ to remove any bacteria that still adhered to the discs. This method is known to effectively remove biomaterial-adherent bacteria [17]. The vortexed solutions were serially diluted 10 -fold, and the final three dilutions were plated in triplicate onto TSA and incubated at $37^{\circ} \mathrm{C}$ for $24 \mathrm{~h}$. The TSA culture plates containing colonies were photographed.

2.4.3. CLSM Assay. After the titanium discs were washed three times with PBS to remove any nonadherent bacteria, they were stained with $500 \mu \mathrm{L}$ of fluorescent dye (LIVE/DEAD BacLight Bacterial Viability Kit, Molecular Probes, L13152) in the dark at room temperature for $15 \mathrm{~min}$ and were then analyzed with CLSM (Leica TCS SP2; Leica Microsystems, Heidelberg, Germany). The LIVE/DEAD kit contains two kinds of fluorescent dye. Viable and nonviable cells can be distinguished under a fluorescence microscope because SYTO $^{\text {тм }} 9$ dye causes viable bacteria with intact cell membranes to display green fluorescence, whereas propidium iodide causes nonviable bacteria with damaged membranes to display red fluorescence. Images were acquired from random positions on the disc surfaces.

2.5. Effect of Incubation Time on Mature Biofilm. To observe the effects of SHJD on the mature biofilms on titanium surfaces after different periods of incubation $(0,4,8,12,16,20$, and $24 \mathrm{~h}$ ), $2 \mathrm{~mL}$ of $\mathrm{NaCl}$ and $\mathrm{NaCl}$ containing a different concentration of SHJD were transferred into each well of a 24well culture plate containing a titanium disc covered with mature biofilm. Six replicate wells were analyzed for each strain at each time point. The plates were placed under aerobic conditions with a low shaking rate $(100 \mathrm{rpm})$ for $24 \mathrm{~h}$. At the specified time points, the culture medium was removed from the wells, and the titanium discs were gently washed three times with PBS and transferred to another 24-well plate. The bacterial biofilms on the titanium surfaces were analyzed with the following methods.

2.5.1. The Spread Plate Method. At each time point, the titanium discs whose surfaces were covered with a residual biofilm were transferred to a $10 \mathrm{~mL}$ glass tube containing $1 \mathrm{~mL}$ of PBS. The tubes were placed in an ultrasonic bath, and the bacteria or biofilm on the discs were dislodged as described above. The vortexed solutions were serially diluted 10-fold, plated on TSA, and incubated at $37^{\circ} \mathrm{C}$ for $24 \mathrm{~h}$. The numbers of colonies formed by the surviving bacteria were 


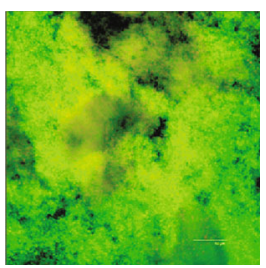

(a)

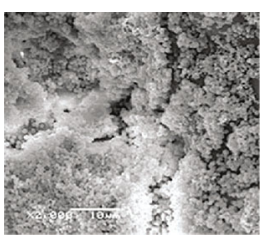

(d)

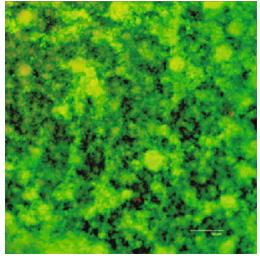

(b)

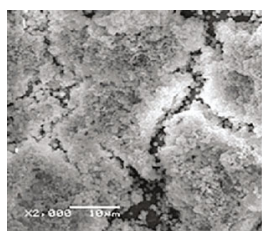

(e)

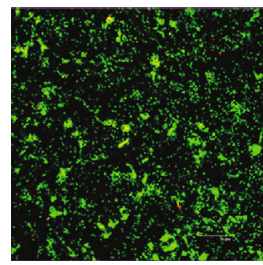

(c)

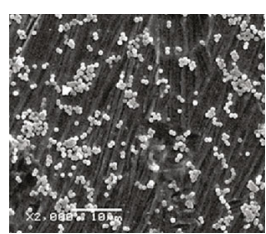

(f)

Figure 1: Mature biofilms on titanium surfaces after incubation for $24 \mathrm{~h}$ in TSB. (a) ATCC 43300 CLSM, (b) ATCC 35984 CLSM, (c) ATCC 12228 CLSM, (d) ATCC 43300 SEM, (e) ATCC 35984 SEM, and (f) ATCC 12228 SEM.

counted, and the numbers of bacteria in the biofilms were calculated and expressed relative to the titanium surface area $\left(\mathrm{CFUs} / \mathrm{mm}^{2}\right)$. The number of bacteria at $0 \mathrm{~h}$ was used as the initial value for each test solution, and the numbers at $4,8,12$, 16,20 , and $24 \mathrm{~h}$ were used as the values after different incubation periods. The antibiofilm efficiency of SHJD was calculated after different periods of incubation with Eq. (1), according to a previous report [18]:

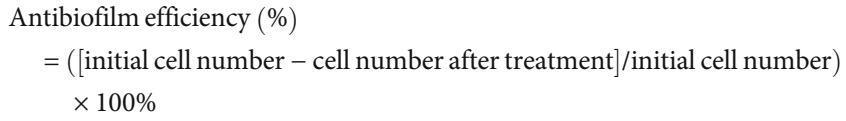

2.5.2. SEM Assay. At each time point, the titanium discs whose surfaces were covered with biofilm were transferred into another 24-well plate and gently washed three times with PBS to remove any nonadherent bacteria. The discs were prepared for SEM with standard procedures. The biofilms on the titanium surfaces were fixed with $2.5 \%$ glutaraldehyde for $2 \mathrm{~h}$ at $4^{\circ} \mathrm{C}$, washed three times with cacodylate buffer, and dehydrated through a graded series of ethanol solutions $(25 \%$, $50 \%, 75 \%, 95 \%$, and 100\%). The samples were then freeze dried, sputter coated with gold, and observed with SEM (Joel JSM-6310LV, JEOL Ltd, Tokyo, Japan).

2.6. Statistical Analysis. All the experiments were performed in triplicate. The results are presented as means \pm standard deviations (SD). The results for each bacterial strain were tested with one-way analysis of variance (ANOVA) with Tukey's multiple comparison test. The differences observed between samples were considered to be significant at $P<$ 0.05 . All analyses were performed with the SPSS 19.0 analysis software (SPSS Inc., Chicago, IL, USA).

\section{Results}

3.1. Morphology of Mature Biofilm. The CLSM images of the mature biofilms of each bacterial strain on the titanium surfaces are shown in Figure 1. After culture in TSB for $72 \mathrm{~h}$,
ATCC 43300 and ATCC 35984 produced numerous bacterial colonies, and the confluence of these colonies formed dense biofilms displaying high-intensity green fluorescence on the titanium surfaces (Figures 1(a) and 1(b)). In contrast, ATCC 12228 showed only scattered clustered colonies displaying low-intensity green fluorescence, indicating no biofilm formation (Figure 1(c)). SEM images showed that the biofilms of ATCC 43300 and ATCC 35984 were composed of many multilayered bacterial colonies, and the colonies clustered together to form dense structures (Figures 1(d) and 1(e)). However, the number and cluster size of the bacterial colonies of ATCC 12228 were dramatically lower than those of the other two strains, and the bacteria clustered as scattered colonies, suggesting that no mature biofilm formed (Figure 1(f)).

In the $\mathrm{CV}$ staining analysis, the $\mathrm{OD}_{492}$ value for $A T C C$ 12228 was $0.11 \pm 0.02$, which was significantly lower than that for ATCC $35984(0.42 \pm 0.05)$ or ATCC 43300 $(0.41 \pm 0.03)(P<0.05)$. The $\mathrm{OD}_{492}$ value for ATCC 12228 was <0.120, and those for ATCC 35984 and ATCC 43300 were both $>0.240$, suggesting that ATCC 12228 is a nonbiofilm-producing strain and that both ATCC 35984 and ATCC 43300 are strong biofilm-producing strains [16, 19].

\subsection{SHJD Weakens Mature Biofilms on Titanium Surfaces}

3.2.1. Quantitative Assay of CV Staining. As seen in Figure 2, the $\mathrm{OD}_{492}$ values for the SHJD (DC:1/4), SHJD (DC:1/2), and SHJD (DC:1/1) groups were significantly lower than those for the control and SHJD (DC:1/8) groups for all three bacterial strains $(P<0.05)$. In the control and SHJD (DC:1/8) groups, no significant differences in the $\mathrm{OD}_{492}$ values were detected $(P>0.05)$. However, the $\mathrm{OD}_{492}$ values differed significantly among the SHJD (DC:1/8), SHJD (DC:1/4), SHJD (DC:1/2), and SHJD (DC:1/1) groups $(P<0.05)$. For ATCC 12228 , the $\mathrm{OD}_{492}$ value for each group was $<0.120$ because it is a non-biofilm-forming strain. However, for both strong biofilm-producing strains, ATCC 35984 and ATCC 43300, incubation with SHJD (DC:1/4), SHJD (DC:1/2), or SHJD (DC:1/1) caused a significant reduction in the $\mathrm{OD}_{492}$ value. 


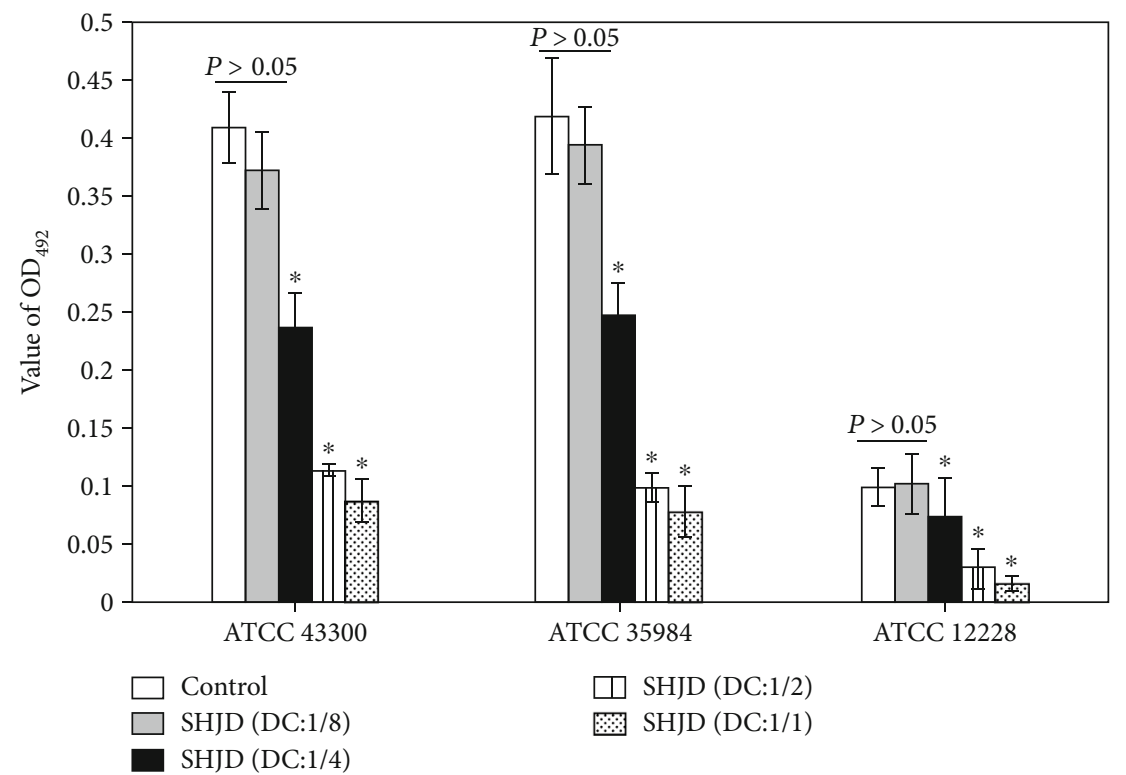

FIGURE 2: $\mathrm{OD}_{492}$ values for mature biofilms on titanium surfaces according to SHJD concentration. For each strain, ${ }^{*} P<0.05$, compared with the control and SHJD (DC:1/8) groups.

The SHJD (DC:1/2) and SHJD (DC:1/1) groups had the lowest $\mathrm{OD}_{492}$ values $(<0.120)$, and the SHJD (DC:1/4) group had the moderate values $(<0.240)$ [19].

3.2.2. Results of the Spread Plate Method. The colonies grown from the surviving bacteria in the residual biofilms on the titanium surfaces were counted on TSA. As shown in Figure 3, the number of viable bacterial colonies in the SHJD (DC:1/1), SHJD (DC:1/2), and SHJD (DC:1/4) groups were lower than those in the control and SHJD (DC:1/8) groups. As the concentration of SHJD decreased, the number of the surviving colonies clearly increased, indicating that the SHJD concentration dependently eradicated the mature biofilms of strains ATCC 43300 and ATCC 35984 and the adhesion of strain ATCC 12228 on a titanium surface.

3.2.3. CLSM Assay. As can be seen from Figure 4, the mature ATCC 43300 and ATCC 35984 biofilms on titanium surfaces displayed different fluorescence intensities on CLSM after they were incubated in different culture media. In the control and SHJD (DC:1/8) groups, the high-intensity green fluorescence on the titanium surfaces indicated that large numbers of bacterial colonies had accumulated and formed bacterial biofilms. However, the fluorescence intensity of the biofilms decreased in all the groups treated with SHJD. In the SHJD (DC:1/2) and SHJD (DC:1/1) groups, only scattered colonies displayed green fluorescence, indicating that the biofilms had been disrupted. In the SHJD (DC:1/4) group, the intensity of fluorescence was lower, suggesting the presence of some residual biofilm, although it was weakened. Only colony adhesion with scattered low-intensity green fluorescence was observed for strain ATCC 12228 , because it is inherently unable to form a biofilm. In the SHJD-treated groups, the scattered green fluorescence decreased as the concentration of SHJD increased.

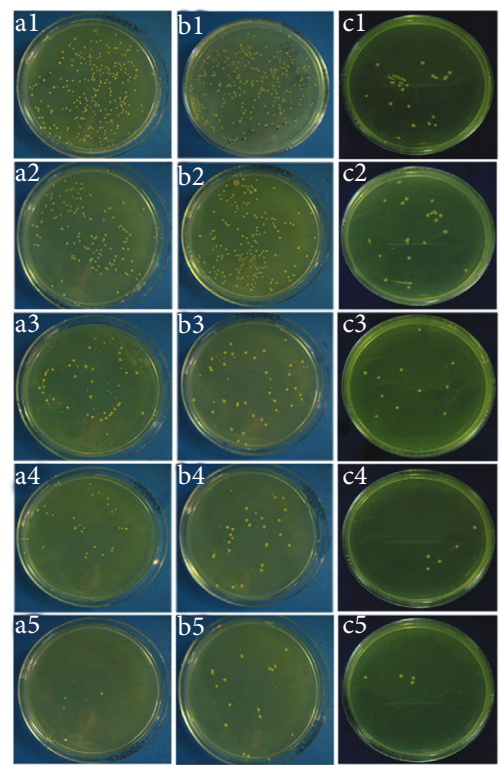

FIgURE 3: Images of bacterial growth after incubation on TSA for 24 h. (1) Control, (2) SHJD (DC:1/8), (3) SHJD (DC:1/4), (4) SHJD (DC:1/2), and (5) SHJD (DC:1/1) against (a) ATCC 43300, (b) ATCC 35984, and (c) ATCC 12228.

\subsection{Different Incubation Times Affect Mature Biofilms}

3.3.1. Antibiofilm Assay. The antibiofilm efficacy of SHJD, measured as the number of surviving bacterial colonies after different incubation times, was calculated with Equation (1) and is shown in Figure 5. These results show that the antibiofilm activities of SHJD at dilutions of $1 / 1$ and $1 / 2$ against each strain were significantly higher than those of the other dilutions at $8-24 \mathrm{~h}(P<0.01)$. The antibiofilm efficacy of the initial SHJD solution diluted $1 / 4$ was also higher than those of control solution and SHJD (DC:1/8) at $8-24 \mathrm{~h}(P<0.05)$. 


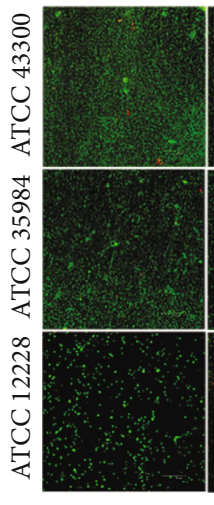

(a)

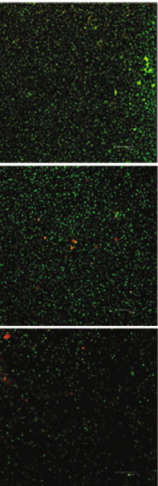

(b)

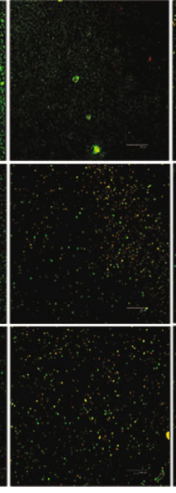

(c)

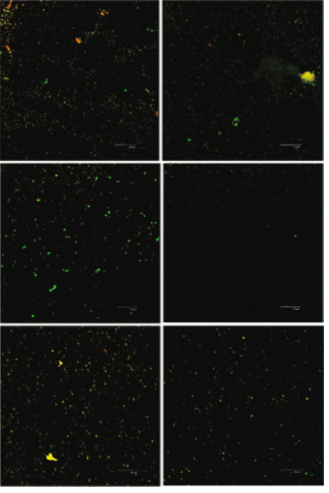

(d)

(e)

Figure 4: CLSM images of biofilms after incubation for $24 \mathrm{~h}$. (a) Control, (b) SHJD (DC:1/8), (c) SHJD (DC:1/4), (d) SHJD (DC:1/2), and (e) SHJD (DC:1/1) groups.

From 12 to $24 \mathrm{~h}$, no differences were observed between SHJD (DC:1/1) and SHJD (DC:1/2) against ATCC 35984, or among SHJD (DC:1/1), SHJD (DC:1/2), and SHJD (DC:1/4) against ATCC $12228 \quad(P>0.05)$. Significant differences were observed between SHJD (DC:1/1) and SHJD (DC:1/2) against ATCC $43300(P<0.05)$. There were no statistically significant differences in the antibiofilm efficacies of the control solution and SHJD (DC:1/8) against any strain at $4-24 \mathrm{~h}$ $(P>0.05)$. At $24 \mathrm{~h}$, the antibacterial efficacy of SHJD (DC:1/2) against ATCC 43300, ATCC 35984, and ATCC 12228 was $67.3 \%, 90.3 \%$, and $99.8 \%$, respectively, and that of SHJD (DC:1/1) against ATCC 43300, ATCC 35984, and ATCC 12228 was $81.8 \%, 95.4 \%$, and $99.9 \%$, respectively.

3.3.2. SEM Assay. The SEM images in Figure 6 show the biofilm morphologies of the three bacterial strains in the SHJD (DC:1/2) group on the titanium surface after different periods of incubation. For strains ATCC 43300 and ATCC 35984 , biofilms with dense multilayered bacterial colonies were observed at 0 and $4 \mathrm{~h}$. At $8-24 \mathrm{~h}$, the biofilms gradually disintegrated as the incubation period increased. The bacterial colonies on the titanium surfaces in the SHJD (DC:1/1) and (DC:1/2) groups were scattered and single, clearly indicating that the biofilm was disrupted. In the SHJD (DC:1/4) group, many residual bacterial colonies still clustered together, forming a moderate biofilm on the titanium surface. For strain ATCC 12228, only colony adhesion was observed after each period of incubation because it has no inherent biofilm-forming capacity.

\section{Discussion}

Infections caused by MDR Staphylococcus strains have now become a serious global public health concern because morbidity and the risk of mortality are increased in patients, and the economic burden has increased for health insurance systems $[20,21]$. Studies have shown that $80 \%$ of clinical bacterial infections are caused by bacterial biofilms, including infections associated with medical devices, such as orthopedic implants, catheters, and heart valves [22]. The leading pathogens causing clinical orthopedic implant-related infec- tions are S. aureus and S. epidermidis [22]. Biofilms are structured multicellular communities of sessile bacterial cells embedded within a self-produced matrix that is made up of proteins, DNA, and polysaccharides, which together act as physical and physiological barriers to antimicrobial agents [23]. The establishment of bacterial biofilms proceeds in two phases: the primary attachment of bacterial cells on a biomaterial is followed by the accumulation of bacteria in multiple layers and glycocalyx formation, generating a mature biofilm [5]. Despite many improvements in the management of orthopedic-biofilm-related infections, treatment failure still remains a major clinical problem because within mature biofilms, the bacteria are resistant to both antibiotics and the host immune system [24].

The long-term administration of effective systemic antibiotics is usually required to treat chronic orthopedicbiofilm-related infections [22]. However, the bacteria in biofilms are in a metabolically quiescent state, and the success of classical culturing to identify them may be as low as 30\% [25]. Even if the biofilm bacteria are detected, a drug sensitivity test usually shows that the orthopedic-biofilm-related infection is caused by MDR Staphylococcus, for which vancomycin is the preferred treatment [6]. However, vancomycin administered systemically has poor bone permeability, with only $10 \%$ of the bone/serum ratio of the drug reaching the bone tissue, and an even smaller amount penetrating the bone tissue at the site of osteomyelitis [26]. Vancomycin kills bacteria by targeting their cell walls, whereas the bacteria in biofilms are protected by the biofilm membranes and are in an inactive and low metabolic state. This also greatly reduces the efficacy of vancomycin [27]. In vitro experiments have shown that vancomycin can penetrate biofilms and kill most (but not all) of the staphylococci growing in the biofilm within $24 \mathrm{~h}$ [28]. However, the concentrations tested in in vitro experiments range from $50 \mathrm{mg} / \mathrm{L}$ to $1000 \mathrm{mg} / \mathrm{L}$, and it is not possible to achieve this concentration of the antibiotic with parenteral administration because vancomycininduced nephrotoxicity can occur after conventional and high doses of the antibiotic [29]. Widespread abuse has also resulted in the emergence of a vancomycin-resistant strain of Staphylococcus [7]. Therefore, it is urgent that a new 


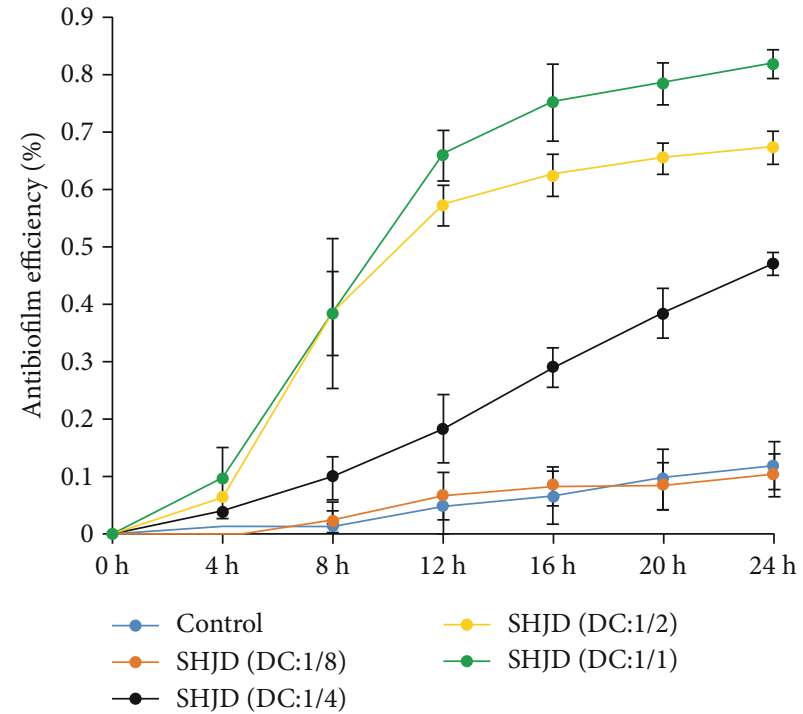

(a)

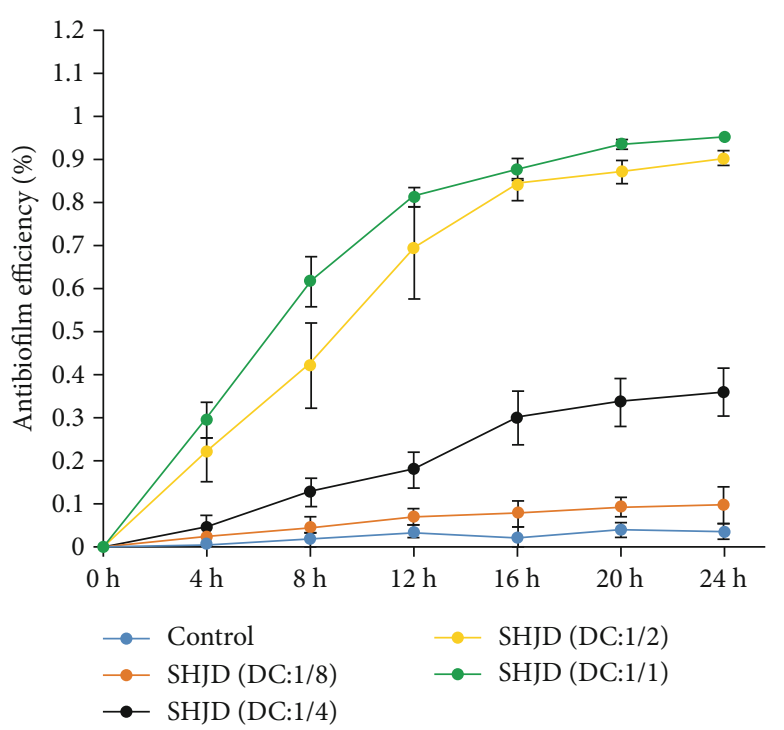

(b)

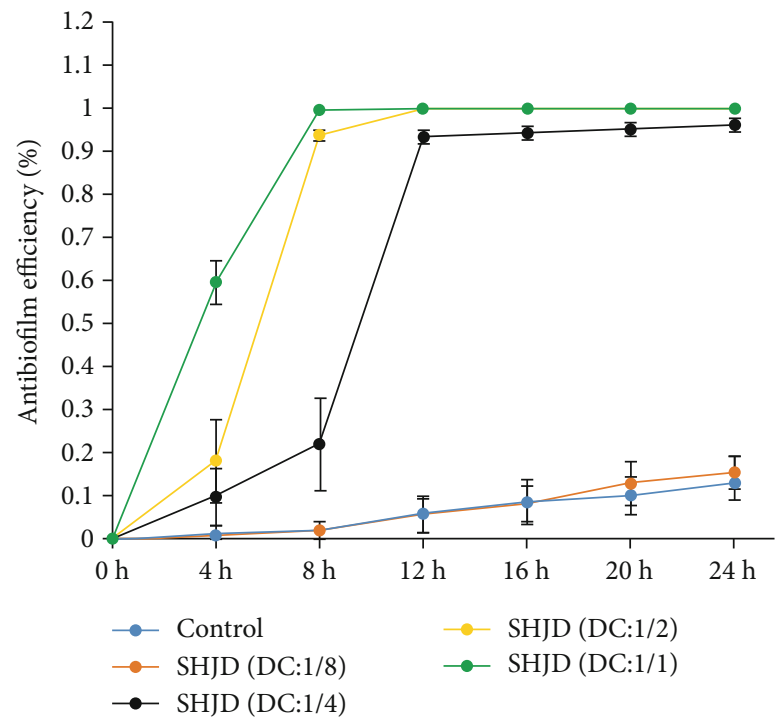

(c)

Figure 5: Effects of SHJD against mature biofilms after different periods of incubation. (a) ATCC 43300, (b) ATCC 35984, and (c) ATCC 12228. Among the SHJD-treated groups, SHJD exerted antibiofilm activity against ATCC 43300 and ATCC 34984, and antibacterialadhesion activity against ATCC 12228 .

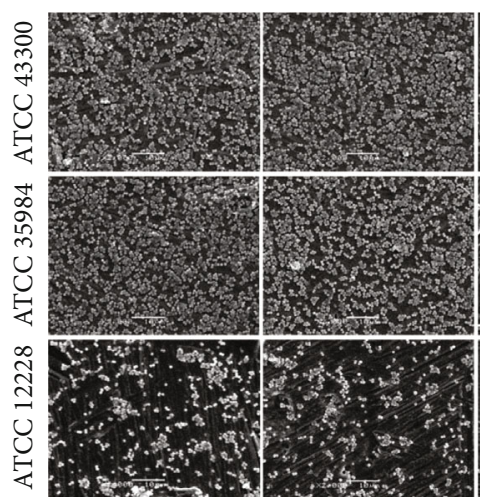

(a)

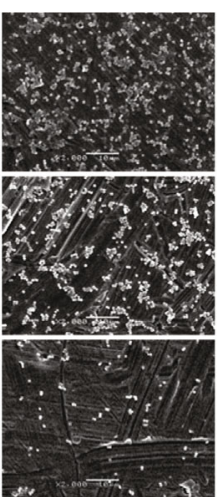

(c)

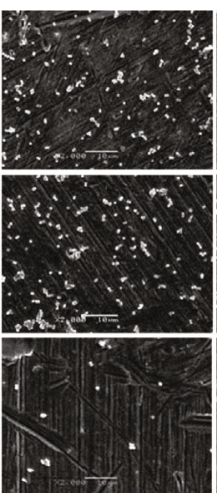

(d)

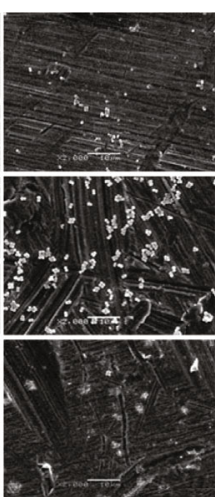

(e)

FIGURE 6: SEM images of biofilms after different periods of incubation with SHJD (DC:1/2). (a) $0 \mathrm{~h}$, (b) $4 \mathrm{~h}$, (c) $8 \mathrm{~h}$, (d) $12 \mathrm{~h}$, and (e) $24 \mathrm{~h}$. 
antibiofilm strategy be developed to overcome the drug resistance of bacteria and to combat the biofilm infections caused by the MDR bacteria. One of these strategies has been identified using a medicinal plant-derived product or herbal extract of TCM.

There is increasing interest in the use of medicinal plantderived compounds as alternative antibacterial agents for infectious diseases [30-35]. However, most basic and clinical research has focused on the efficacy of the bioactive compounds isolated from plants against virulent pathogens, and few studies have examined the anti-infection activities of compound preparations [8-10, 32-35]. TCM has been used in the Chinese population to treat various infectious diseases for thousands of years, and many preparations have been shown to be therapeutically effective [8-10, 12-14]. The value of externally applied combination TCM preparations for the prevention and treatment of orthopedic infections has also been confirmed [12-14]. We have routinely used SHJD extract to directly soak and wash the infected wounds with bone and implant exposure in order to effectively destroy the bacterial biofilm and control the local infections of the extremities caused by multiple-antibiotic-resistant bacteria. The infected wound was soaked and washed for $30 \mathrm{~min}$ in SHJD extract twice a day, and after treatment for mean 3 weeks, we have found that local infections of the limbs are clearly controlled, the tissue swelling subsides, secretions and necrotic tissue are reduced, and the granulation tissue grows well, which provide better tissue conditions for wound healing. Modern medicinal research has shown that the heat-clearing and detoxifying herbs that comprise SHJD exert broad-spectrum antibacterial and bacteriostatic effects without bacterial resistance when they are applied in combination. Semen persicae, Boswellia sacra, and Commiphora myrrha promote blood circulation and the flow of qi, reduce swelling, and induce granulation, thus improving the local microcirculation and promoting tissue regeneration. The clinical application of SHJD has both antibacterial and anti-inflammatory roles, improving the local blood circulation to the wound and enhancing the local immunity, thus eliminating the infection and promoting wound healing [12-14].

To investigate the in vitro effects of the antibiofilm properties of SHJD in this study, the maturity of the biofilm was considered. Biofilms that have developed for only $24 \mathrm{~h}$ are always immature, and antibiotics can still kill the bacteria in these biofilms [36]. On the contrary, the metabolism of the bacteria in thick, multilayered, mature biofilms is relatively inactive, which allows them to tolerate extremely high antibiotic concentrations, so the eradication of these biofilms with antibiotics may be very difficult $[36,37]$. Orthopedic implant-related infections are often detected after a few weeks in clinical practice, during which time the bacterial biofilm has developed into a mature form, with multiple layers and glycocalyx, and is therefore resistant to antibiotics. Based on these findings, we used a biofilm that had been incubated for 3 days to reflect the actual situation of mature biofilms [37]. ATCC 35984 and ATCC 43300 are powerful biofilm-forming strains, whereas ATCC 12228 is a nonbiofilm-forming strain, and these were selected to test the weakening or eradication of mature biofilms by SHJD. We also examined the morphological changes in the biofilms that occurred under low-speed horizontal shaking, which simulated the possible flow of the tissue fluid in the infected wound. The quantitative and qualitative analyses in this study demonstrated that SHJD weakens and disrupts the mature biofilms formed by the antibiotic-resistant Staphylococcus on titanium surfaces. This effect tended to increase as either the concentration of SHJD or the period of incubation increased. Specifically, the mature biofilms of ATCC 43300 and ATCC 35984 on the titanium surfaces clearly disintegrated after incubation for $12-24 \mathrm{~h}$ with the initial SHJD solution (DC:1/1) or (DC:1/2), producing only scattered adherent bacterial colonies. With the SHJD (DC:1/4) treatment, although many residual bacterial colonies still clustered together, forming a weak biofilm on the titanium surfaces, the effect was greater than that in the SHJD (DC:1/8) group, in which the biofilm was only slightly weakened compared with that in the control group. Only colony adhesion was observed for strain ATCC 12228, but in the SHJD groups, the number of bacterial colonies decreased as the concentration of SHJD or the culture period increased.

Biofilm-related infections can be eradicated by antibiofilm agents that weaken or destroy the mature biofilm rendering cells to be susceptible to antibiotics [34]. In this study, the ability of SHJD to effectively weaken or disrupt mature biofilms suggests that it may increase the penetration of antibiotics in the biofilm and reduce bacteria antibiotic tolerance. This hypothesis needs to be validated by the synergistic effect of SHJD combined with conventional antibiotics on the mature bacterial biofilms. In the light of the findings of the current study, it is also important for further studies to clarify the mechanism of action of SHJD as an antibiofilm agent and to investigate the efficacy of SHJD to weaken the orthopedic-biofilm-related infections caused by MDR Staphylococcus in vivo.

\section{Conclusions}

In summary, this study demonstrates the SHJD decoction has antibiofilm activity against Staphylococcus strains with multidrug resistance, and it can weaken or disrupt already formed mature biofilms on the titanium surface with a dilution concentration-dependent and incubation time-dependent manner. It may be useful in the development of antibacterial agents to treat orthopedic-biofilm-related infections.

\section{Abbreviations}

SHJD: Sanhuang Jiedu decoction

TCM: Traditional Chinese medicine

MIC: Minimum inhibitory concentration

TSB: Tryptic soy broth

TSA: Tryptone soy agar

CV: Crystal violet

SEM: Scanning electron microscopy

CLSM: Confocal laser scanning microscopy

MRSE: Multidrug-resistant Staphylococcus epidermidis

MRSA: Multidrug-resistant Staphylococcus aureus 
MDR: Multidrug-resistance

Rpm: Revolutions per minute

CFUs: Colony-forming units

DC: Dilution concentrations

PBS: $\quad$ Phosphate-buffered saline

PI: $\quad$ Propidium iodide

OD: Optical density.

\section{Data Availability}

The data used to support the findings of this study are available from the corresponding author upon request.

\section{Conflicts of Interest}

The authors declare that they have no conflicts of interest.

\section{Authors' Contributions}

SEZ drafted the manuscript. SEZ and XW acquired and analyzed the data. XTS helped interpret the results. HLT designed and planned the study. All authors revised and reviewed this work, and all authors gave their final approval of the submitted manuscript.

\section{Acknowledgments}

This work was supported by the NSFC-Henan Joint Fund (No.U1804189).

\section{References}

[1] W. J. Metsemakers, R. Kuehl, T. F. Moriarty et al., "Infection after fracture fixation: current surgical and microbiological concepts," Injury, vol. 49, no. 3, pp. 511-522, 2018.

[2] B. Höing, L. Kirchhoff, J. Arnolds et al., "Bioactive glass granules inhibit mature bacterial biofilms on the surfaces of cochlear implants," Otology \& Neurotology, vol. 39, no. 10, pp. e985-e991, 2018.

[3] N. Høiby, T. Bjarnsholt, M. Givskov, S. Molin, and O. Ciofu, "Antibiotic resistance of bacterial biofilms," International Journal of Antimicrobial Agents, vol. 35, no. 4, pp. 322-332, 2010.

[4] J. G. Leid, M. E. Shirtliff, J. W. Costerton, and P. Stoodley, "Human leukocytes adhere to, penetrate, and respond to Staphylococcus aureus biofilms," Infection and Immunity, vol. 70, no. 11, pp. 6339-6345, 2002.

[5] M. K. Suresh, R. Biswas, and L. Biswas, "An update on recent developments in the prevention and treatment of Staphylococcus aureus biofilms," International Journal of Medical Microbiology, vol. 309, no. 1, pp. 1-12, 2019.

[6] H. S. Fraimow, "Systemic antimicrobial therapy in osteomyelitis," Seminars in Plastic Surgery, vol. 23, no. 2, pp. 90-99, 2009.

[7] B. P. Howden, J. K. Davies, P. D. R. Johnson, T. P. Stinear, and M. L. Grayson, "Reduced vancomycin susceptibility in Staphylococcus aureus, including vancomycin-intermediate and heterogeneous vancomycin-intermediate strains: resistance mechanisms, laboratory detection, and clinical implications," Clinical Microbiology Reviews, vol. 23, no. 1, pp. 99-139, 2010.
[8] S. T. Micek, "Alternatives to vancomycin for the treatment of Methicillin-Resistant Staphylococcus aureus infections," Clinical Infectious Diseases, vol. 45, Supplement_3, pp. S184S190, 2007.

[9] W. Yang, J. Liu, B. Blažeković et al., "In vitro antibacterial effects of Tanreqing injection combined with vancomycin or linezolid against methicillin-resistant Staphylococcus aureus," BMC Complementary and Alternative Medicin, vol. 18, no. 1, pp. 169-179, 2018.

[10] A. Flower, L.-Q. Wang, G. Lewith, J. P. Liu, and Q. Li, “Chinese herbal medicine for treating recurrent urinary tract infections in women," Cochrane Database of Systematic Reviews, vol. 3, pp. 1-12, 2013.

[11] X. Tong, A. Li, Z. Zhang et al., "TCM treatment of infectious atypical pneumonia-a report of 16 cases," Journal of Traditional Chinese Medicine, vol. 24, no. 4, pp. 266-269, 2004.

[12] C. S. Cheng, Y. Q. Jin, and Z. Z. Cheng, "Clinical observation on 267 patients with leg osteocutaneous defect due to wound infection treated by integrated traditional and Western medicine," World Journal of Integrated Traditional and Western Medicine, vol. 2, no. 9, pp. 535-538, 2007.

[13] F. Feng and C. S. Cheng, "Integration of TCM and western medicine for the treatment of traumatic osteomyelitis combined with osseous defect," China Journal of Orthopaedics and Traumatology, vol. 13, no. 11, pp. 643-644, 2000.

[14] J. N. Yao, C. S. Cheng, and Z. G. Zhao, "Reverse anterolateral femoral flap combined with traditional Chinese medicine fumigation in the treatment of proximal tibial bone-skin defect with infection," Chinese Journal of Traditional Medical Traumatology \& Orthopedics, vol. 22, no. 6, pp. 19-20, 2014.

[15] M. Á. Pacha-Olivenza, A. Rodríguez-Cano, M. L. GonzálezMartín, and A. M. Gallardo-Moreno, "Kinetic of adhesion ofS. epidermidiswith different EPS production on Ti6Al4V surfaces," Biomed Research International, vol. 2019, Article ID 1437806, 8 pages, 2019.

[16] H. Tan, Z. Peng, Q. Li, X. Xu, S. Guo, and T. Tang, "The use of quaternised chitosan-loaded PMMA to inhibit biofilm formation and downregulate the virulence-associated gene expression of antibiotic-resistant staphylococcus," Biomaterials, vol. 33, no. 2, pp. 365-377, 2012.

[17] G. Bjerkan, E. Wits $\varnothing$, and K. Bergh, "Sonication is superior to scraping for retrieval of bacteria in biofilm on titanium and steel surfaces in vitro," Acta Orthopaedica, vol. 80, no. 2, pp. 245-250, 2009.

[18] Z. X. Peng, L. Wang, L. du, S. R. Guo, X. Q. Wang, and T. T. Tang, "Adjustment of the antibacterial activity and biocompatibility of hydroxypropyltrimethyl ammonium chloride chitosan by varying the degree of substitution of quaternary ammonium," Carbohydrate Polymers, vol. 81, no. 2, pp. 275$283,2010$.

[19] T. Mathur, S. Singhal, S. Khan, D. J. Upadhyay, T. Fatma, and A. Rattan, "Detection of biofilm formation among the clinical isolates of Staphylococci: an evaluation of three different screening methods," Indian Journal of Medical Microbiology, vol. 24, no. 1, pp. 25-29, 2006.

[20] World Health Organization, Antimicrobial Resistance: Global report on Surveillance 2014, WHO, Geneva, 2014.

[21] N. Kumari, S. Singh, V. Kumari, S. Kumar, V. Kumar, and A. Kumar, "Ouabain potentiates the antimicrobial activity of aminoglycosides against Staphylococcus aureus," BMC Complementary and Alternative Medicine, vol. 19, no. 1, p. 119, 2019. 
[22] C. R. Arciola, D. Campoccia, and L. Montanaro, "Implant infections: adhesion, biofilm formation and immune evasion," Nature Reviews Microbiology, vol. 16, no. 7, pp. 397-409, 2018.

[23] S. J. McConoughey, R. Howlin, J. F. Granger et al., "Biofilms in periprosthetic orthopedic infections," Future Microbiology, vol. 9, no. 8, pp. 987-1007, 2014.

[24] X. M. Liu, Y. Zhang, F. Chen et al., "Prevention of orthopedic device-associated osteomyelitis using oxacillin-containing biomineral-binding liposomes," Pharmaceutical Research, vol. 29, no. 11, pp. 3169-3179, 2012.

[25] L. Hall-Stoodley, F. Z. Hu, A. Gieseke et al., "Direct detection of bacterial biofilms on the middle-ear mucosa of children with chronic otitis media," The Journal of the American Medical Association, vol. 296, no. 2, pp. 202-211, 2006.

[26] A. L. Graziani, L. A. Lawson, G. A. Gibson, M. A. Steinberg, and R. R. MacGregor, "Vancomycin concentrations in infected and noninfected human bone," Antimicrobial Agents and Chemotherapy, vol. 32, no. 9, pp. 1320-1322, 1988.

[27] A. P. Cardile, C. J. Sanchez, M. E. Samberg et al., "Human plasma enhances the expression of Staphylococcal microbial surface components recognizing adhesive matrix molecules promoting biofifilm formation and increases antimicrobial tolerance in vitro," BMC Research Notes, vol. 457, pp. 1-9, 2014.

[28] E. Ampe, B. Delaere, J. D. Hecq, P. M. Tulkens, and Y. Glupczynski, "Implementation of a protocol for administration of vancomycin by continuous infusion: pharmacokinetic, pharmacodynamic and toxicological aspects," International Journal of Antimicrobial Agents, vol. 41, no. 5, pp. 439-446, 2013.

[29] A. Ghimire, J. D. Skelly, and J. Song, "Micrococcal-nucleasetriggered on-demand release of vancomycin from intramedullary implant coating eradicates Staphylococcus aureus infection in mouse femoral canals," ACS Central Science, vol. 5, no. 12, pp. 1929-1936, 2019.

[30] Y. Ma, Y. Wang, H. Zhang et al., "Antimicrobial mechanism of strictinin isomers extracted from the root of Rosa roxburghii Tratt (Ci Li Gen)," Journal of Ethnopharmacology, vol. 250, article 112498, 2020.

[31] J. N. Eloff, "Avoiding pitfalls in determining antimicrobial activity of plant extracts and publishing the results," $B M C$ Complementary and Alternative Medicine, vol. 19, no. 106, pp. 1-8, 2019.

[32] J. Chen, C. Tang, R. Zhang et al., "Metabolomics analysis to evaluate the antibacterial activity of the essential oil from the leaves of Cinnamomum camphora (Linn.) Presl," Journal of Ethnopharmacology, vol. 253, article 112652, 2020.

[33] P. N. Kipanga, M. Liu, S. K. Panda et al., "Biofilm inhibiting properties of compounds from the leaves of warburgia ugandensis Sprague subsp ugandensis against Candida and staphylococcal biofilms," Journal of Ethnopharmacology, vol. 248, article 112352, 2020.

[34] W. Mozirandi, D. Tagwireyi, and S. Mukanganyama, "Evaluation of antimicrobial activity of chondrillasterol isolated from Vernonia adoensis (Asteraceae)," BMC Complementary and Alternative Medicine, vol. 19, no. 1, p. 249, 2019.

[35] B. Bhunu, R. Mautsa, and S. Mukanganyama, "Inhibition of biofilm formation in mycobacterium smegmatis by parinari curatellifolia leaf extracts," BMC Complementary and Alternative Medicine, vol. 17, no. 285, pp. 1-10, 2017.
[36] C. Chuard, P. Vaudaux, F. A. Waldvogel, and D. P. Lew, "Susceptibility of Staphylococcus aureus growing on fibronectincoated surfaces to bactericidal antibiotics," Antimicrobial Agents and Chemotherapy, vol. 37, no. 4, pp. 625-632, 1993.

[37] P. S. Stewart, “Antimicrobial tolerance in biofilms," Microbiology Spectrum, vol. 3, no. 3, pp. 1-30, 2015. 